\title{
Rib Segmentation in Chest Radiographs by Support Vector Machine
}

\author{
Wu Haiping \\ School of Computer \\ Shenyang Aerospace University \\ Shenyang 110136, China \\ E-mail: haipingw89@163.com
}

\author{
Zhang Guodong \\ School of Computer \\ Shenyang Aerospace University \\ Shenyang 110136, China \\ E-mail: zhanggd@sau.edu.cn
}

\begin{abstract}
The segmentation of ribs is one of the key problems for computer-aided diagnosis. This paper presented a novel scheme for rib segmentation in chest radiographs. The Gaussian filter was used repeatedly to remove uneven background of chest. Detail images could be obtained by use of the multi-scale wavelet decomposition, and then Gaussian derivative was employed to extract features from the detail images. The rib model for support vector machine (SVM) was established, which was employed to classify ribs. In our study, a method of sample selection with property reduction was used to reduce calculation time. The experimental result shows that proposed scheme can segment ribs in chest radiographs effectively.
\end{abstract}

Keywords-rib segmentation; support vector machine (SVM); wavelet transformation; Gaussian derivative; feature extraction

\section{INTRODUCTION}

The segmentation of ribs is one of the key problems for computer-aided diagnosis. It can be used for effective visualizations of the rib cage. It is also a first step for computerized detection of abnormal bone. And, the segmented ribs can act as reference objects to segment other structures [1-2]. There are several methods for rib segmentation. Yue proposed an algorithm about rib segmentation based on edge detection in 1995 [3]. The algorithm extracted the border of ribs by Hough transform firstly, and then rectified the segmentation result by Snake method. It was difficult to distinguish the upper border and lower border of one rib, because the same rib border can be detected repeatedly. K-means clustering was presented to segment ribs [4]. K-means clustering is an unsupervised learning algorithm which aims at minimizing squared error function. When $\mathrm{K}$ was equal to 2 , images were divided into ribs and background. It could get rough position of each rib. $\mathrm{Xu}$ Chaohui [5] proposed a Gaussian surface threshold to segment ribs in 2004, the original image was filtered with Gaussian template, and the filtered image was used as a threshold surface. This method was simple and efficient. But when ribs were not clear in the image, the result of segmentation was not very satisfied. Wang Xin [6] proposed a detection method for the rib border based on the knowledge of anatomical structures. Borders of a rib were detected by a bi-parabolic model in the position of the rib. But it was difficult to divide the region of each rib, because of the uneven distribution of rib structure. Loog [7] developed a method of Iterated Contextual Pixel Classification(ICPC) for complex segmentation tasks such as ribs. ICPC started from initial rib segmentation by pixel classification and updated it by reclassifying every pixel which was based on the original features and class label information of pixels in the neighborhood of the pixel to be reclassified. The drawback of ICPC was slow calculation speed, so it could not meet real-time processing requirement. Marleen [8] proposed a multi-object segmentation algorithm which based on shape particles. In this algorithm, rib model was deformed, and Gaussian derivative feature was extracted. Then k-nearest neighbor classifier was used as a pixel classifier.

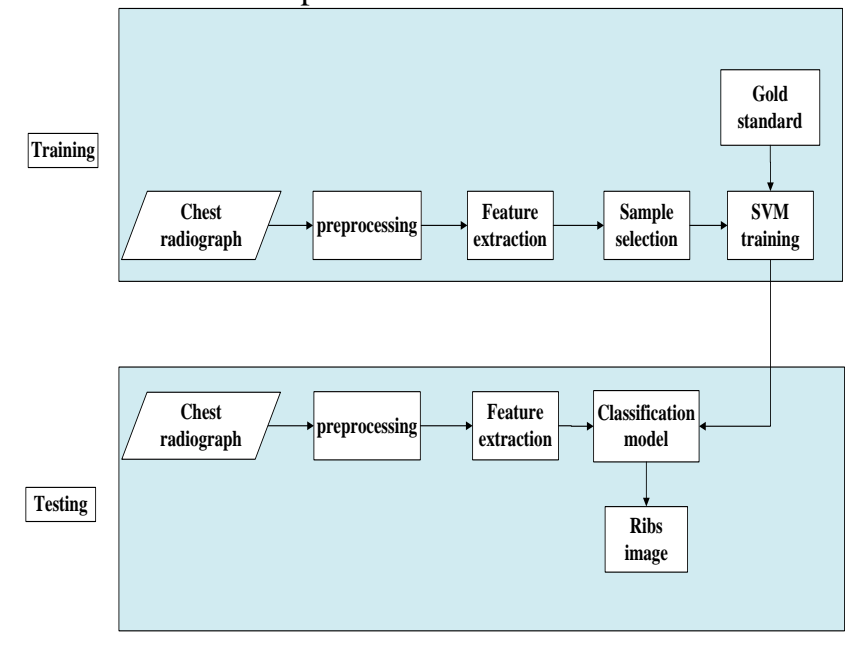

Figure 1. Overall scheme for rib segmentation

This paper proposed a novel method that consisted of preprocessing, feature extraction based on multi-scale wavelet transformation and Gaussian derivative, and classification of pixels by the support vector machine (SVM). In this study, the gold standard was established manually. Fig .1 was the overall scheme of rib segmentation in chest radiograph.

\section{RIB SEGMENTATION BY SVM}

\section{A. Preprocessing}

Preprocessing step is necessary to obtain good results. The $\mathrm{x}$-ray images were noisy and the contrast of images was low. In order to improve the efficiency of processing, 
resample was used to adjust the image size to $512 * 512$ pixels. Gaussian filter is commonly used to remove Gaussian white noise. In 2D, Gaussian function is:

$$
G(x, y)=\frac{1}{2 \pi \sigma^{2}} e^{-\frac{(x-m / 2)^{2}+(y-n / 2)^{2}}{2 \sigma^{2}}}
$$

Where $\mathrm{m}, \mathrm{n}$ are the length and width of the Gaussian template respectively, and $\sigma$ was the standard deviation of the normal distribution. Background image was obtained by smoothing with multiple Gaussian filter:

$$
I=I_{1}-I_{g}+\hat{I}_{g}
$$

$I_{1}$ is the original chest image. $I_{g}$ is the Gaussian blurred image with 400 times. $\hat{I}_{g}$ is the chest image with uniform background.

After the preprocessing, uniform background trend in the lung region can be got, at the same time, the details of rib edge were preserved.

\section{B. Feature extraction}

Extracting effective feature is the basis of established rib model. In this paper, detail images could be obtained by multi-scale wavelet decomposition, and then Gaussian derivative was employed to extract feature from the detail images. Wavelet transform has good localization property in the domain of time and frequency. It is a powerful tool for multi-scale analysis [9]. B-spline function converges to Gaussian function rapidly with the increase of spline order, and its first-order derivative can approach the optimal edge-detection operator. When B-spline wavelet was employed to enhance multi-scale edges, it would achieve a well performance. $h_{k}$ and $g_{k}$ are low-pass filter coefficient and high-pass filter coefficient of third order Bspline wavelet function.

When $k=-2,-1,0,1,2$,

then

$$
\begin{gathered}
h_{k}=\left\{\frac{1}{16}, \frac{1}{4}, \frac{3}{8}, \frac{1}{4}, \frac{1}{16}\right\} \\
g_{k}=\left\{\begin{array}{l}
-2, k=0 \\
2, k=1 \\
0, \text { other }
\end{array}\right.
\end{gathered}
$$

The $h_{k}$ and $g_{k}$ were first applied in one dimension and then in the other. To reduce the overall number of computations, down-sampling was performed in two stages. One scale fast wavelet transform was employed for obtaining 4 sub-images. They were approximate, horizontal, vertical and diagonal detail sub-images. Fig .2 show the result of decomposition of fast wavelet transform. In this paper, cubic wavelet transform was used to extract features.

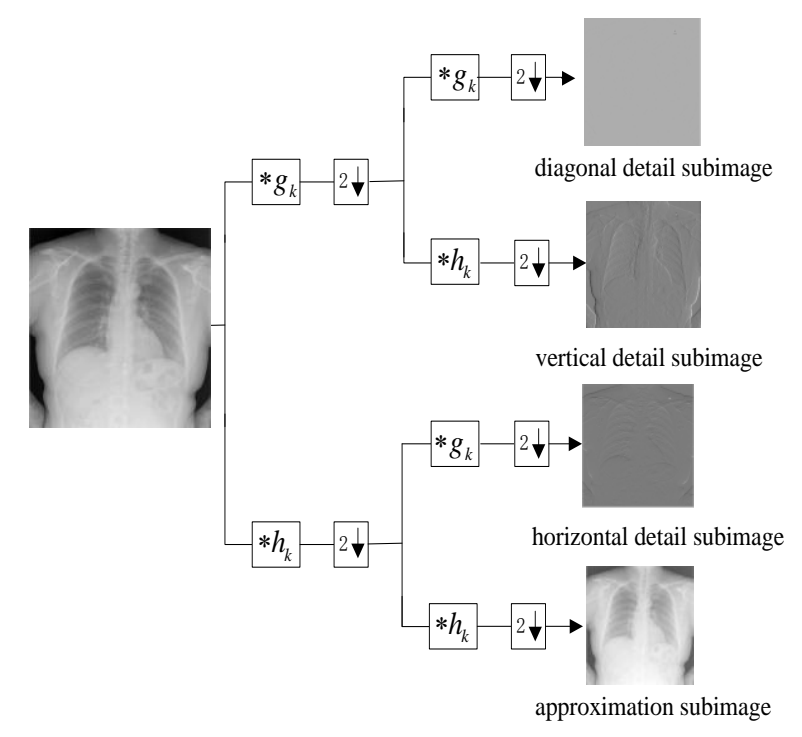

Figure 2. Decomposition of fast wavelet transform

According to Tayor formula, image can be represented by a series of partial derivatives. Gaussian smoothing can reduce noise greatly. Therefore, Gaussian derivative was employed to extract feature from the detail images that got from cubic wavelet transform. Fig .3 show the feature of Gaussian derivative. A is original image with Gaussian smoothing, B is first derivative of $x, \mathrm{C}$ is the first derivative of $y, \mathrm{D}$ is the first derivative of diagonal, $\mathrm{E}$ is the second derivative of $x, \mathrm{~F}$ is the second derivative of $y, \mathrm{G}$ is the mixture derivative, $\mathrm{H}$ is the second derivative of diagonal.

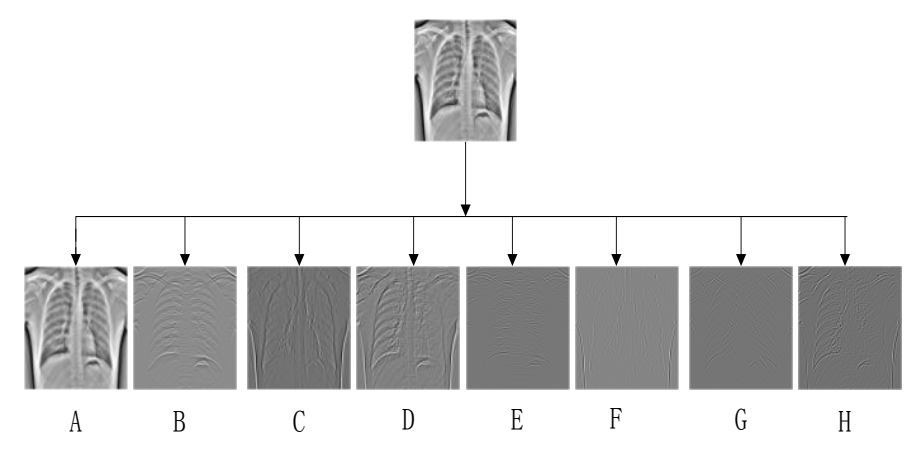

Figure 3. Feature of Gaussian derivative

\section{Building the model of ribs with SVM}

SVM is developed based on the principle of Structural Risk Minimization (SRM) and VC dimension theory [10]. Because of its solid mathematical foundation in statistical learning theory and special idea of classification, SVM has achieved a highly competitive performance in many fields, such as face recognition, voice recognition, and license plate recognition. In this paper, to obtain satisfied performance of pixel classification, nonlinear kernel function is employed to transform input data to a highdimensional feature space. SVM is one of machine 
learning algorithm, which could avoid the problems such as over fitting, curse of dimensionality, and the local extremum. SVM was designed to classify pixels (rib or norib).

The steps of building rib segmentation model can be summarized as follows:

(1) Wavelet transformation and Gaussian derivative were used to extract features images, and then feature images were resized to $512 * 512$ by bilinear interpolation.

(2) Label for each feature vector is necessary, because SVM is a supervised learning model. The gold standard was established manually. The pixels of ribs were set to 1 , and pixels of no- ribs were set to 0 .

(3) The last one was built the rib model with SVM, then the established model was used to predict each pixel of another chest radiograph in test set.

During establishment of a classification model, the large-scale training set slow down training speed [11]. To improve training speed, attribute reduction and sample reduction were used to reduce the training samples in this paper. $\left\{x_{i}, y_{i}\right\}$ is sample set, $x_{i} \in R, y_{i} \in\{1,0\}$, $i=1,2, \cdots l . M_{1}, M_{2}$ represent centers of positive and negative samples respectively. According to geometry distribution of support vectors, the angle between the vector $M_{1} x_{i_{-} 1}$ and the vector $M_{1} M_{2}$ or the angle between the vector $M_{2} x_{i_{-} 2}$ and the vector $M_{1} M_{2}$ are not obtuse angles. Then, according to the cosine of the angle is less than 0 , determine whether the sample is nonsupport vector [12]. To reduce the number of features, the relevance feature vectors were transformed into independent one from each other by using principal component analysis.

\section{EXPERIMENT RESULTS AND ANALYSIS}

\section{A. Experimental Data}

The x-ray images for which we performed the segmentation process made part from a database of images of DICOM type. The image had a size of $2048 \times 2048$ pixels, and we re-sampled the image to reduce the processing time. The re-sampled image had a matrix size of $512 \times 512$ pixels. We have 22 chest radiographs and corresponding manual segmentation mask. 2-fold crossvalidation method was used to evaluate the performance of algorithm.

\section{B. Experimental Result}

Table I shows performance of rib segmentation by extracting Gaussian derivative from chest radiographs directly and Gaussian derivative from the detail images which comes from Wavelet Decomposition. Experimental results show that the latter's accuracy is 5\% higher than the former's accuracy, and the specificity and sensitivity higher than the former, too. Therefore, it is efficient to extract feature of multi-scale wavelet decomposition.

Gaussian derivatives in different directions can show the different variations of gray. Table II shows the performance of partial derivative in different directions. The combination of all features took advantage of intensity's variation, and the best performance was achieved.
TABLE I. PERFORMANCE OF RIB SEGMENTATION WITH DIFFERENT FEATURE

\begin{tabular}{|c|c|c|}
\hline & Gaussian derivative & $\begin{array}{c}\text { Multi-scale wavelet } \\
\text { decomposition and } \\
\text { Gaussian derivative }\end{array}$ \\
\hline Accuracy & 0.80 & 0.85 \\
\hline Specificity & 0.86 & 0.87 \\
\hline Sensitivity & 0.71 & 0.81 \\
\hline
\end{tabular}

In table III, the training time and accuracy were 289 seconds and 0.8546 respectively before reduction. The training time and accuracy were 261 seconds and 0.8573 respectively when the method of sample reduction was used only. The training time and accuracy were 161 seconds and 0.8557 respectively when the method of attributes reduction was used only. The training time and accuracy were 145 seconds and 0.8551 respectively when sample reduction and attributes reduction were combined. The result shows that training time was decreased much without affecting the accuracy. Performance of rib segmentation based on support vector machine shows in Fig .4.

TABLE II. PERFORMANCE OF RIB SEGMENTATION WITH DIFFERENT DIRECTIONS PARTIAL DERIVATIVE

\begin{tabular}{|l|c|}
\hline Feature & Accuracy \\
\hline first derivative of $x$ & 0.82 \\
\hline first derivative of $y$ & 0.69 \\
\hline first derivative of diagonal & 0.79 \\
\hline second derivative of $x$ & 0.80 \\
\hline second derivative of y & 0.67 \\
\hline second derivative of diagonal & 0.78 \\
\hline mixture derivative & 0.72 \\
\hline combination of all features & \\
\hline
\end{tabular}

TABLE III. RESULTS OF SAMPLE SELECTION

\begin{tabular}{|c|c|c|c|c|}
\hline & $\begin{array}{c}\text { Before } \\
\text { reduction }\end{array}$ & $\begin{array}{c}\text { Sample } \\
\text { reduction }\end{array}$ & $\begin{array}{c}\text { Attributes } \\
\text { reduction }\end{array}$ & $\begin{array}{c}\text { After sample } \\
\text { and attributes } \\
\text { reduction }\end{array}$ \\
\hline Time(s) & 289 & 261 & 161 & 145 \\
\hline Accuracy & 0.8546 & 0.8573 & 0.8557 & 0.8551 \\
\hline
\end{tabular}



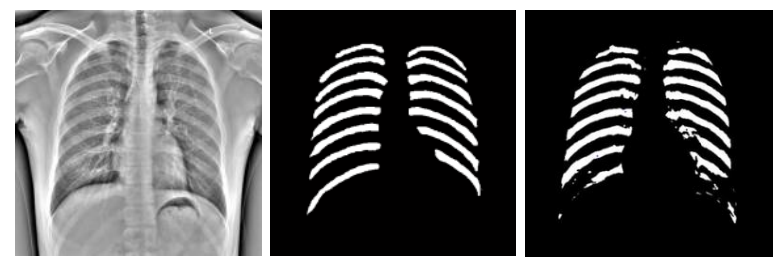

Figure 4. Illustration of the results: (a) Chest image by preprocessing (b) Result of ribs manual segmentation. (c)Result of rib segmentation.

\section{CONCLUSIONS}

We developed a novel scheme to segment ribs, which was based on pixel classification. In order to achieve a high accuracy, multi-scale wavelet transformation and Gaussian derivative were employed to extract local shape characteristic of ribs. Then, the rib model for support vector machine (SVM) was established, which was employed to classify ribs. In our study, a method of sample selection with property reduction was used to reduce calculation time. The experimental result shows that proposed scheme can segment ribs in chest radiographs effectively.

\section{Acknowledgment}

This work was supported in part by National Natural Science Foundation of China (No.61373088, No.61402298), and National Aerospace Science Foundation (No.2013ZE54025).

\section{REFERENCES}

[1] S. Joes, V. Ginneken, "Automatic rib segmentation in CT data", J. Lecture Notes in Computer Science, vol 3117, pp. 193-204, 2004.

[2] C. Cosmin. "segmentation of bone structure in X-ray images using convolutional neural network", J. Advances inElectrical and Computer Engineering, vol 13, 2013.

[3] Z. Yue, A. Goshtasby, VL. Ackerman. "Automatic detection of rib borders in chest radiographs", J, IEEE Transaction on Medical Imaging, vol 14, pp:525-536, 1995.

[4] Xu Chaohui, Zhang Jiwu and Zhang Daobing, "Several Methods for Ribs Segmentation in Di gital Chest Radiograph", China Journal of Image and Graphics, vol.10, pp. 1240-1244, 2004.

[5] X. Chaohui, Z. Lichun and Z. Daobing, "Image Segmentation of Digital Chest Radiographs Based on Gaussian Curve Plane Threshold Method", J, application and project of video technologies, pp. 92-94, 2005.

[6] W. xing, Z.tiange. "Detection of Rib Borders in Chest Radiographs Based on Anatomical Structure Knowledge". Space medicine and medical engineering. vol 06 pp:456-460. 2005.

[7] Loog, M. and Ginneken, B., "Segmentation of the Posterior Ribs in Chest Radiographs Using Iterated Contextual Pixel Classification", IEEE transactions on medical imaging, vol.25, pp.602-611, 2006.

[8] Marleen, M. Nielsen. "Multi-object segmentation using shape particles", J. Information Processing in Medical Imaging, pp. 762773, 2005.

[9] W. Yuping, Cai, Y.L. "Multiscale B-spline Wavelet for Edge Detection". Science in China, vol. 38(4), pp. 499-512.1995.

[10] C. Cortes, Vapnik. "Support-vector network", J, Machine Learning, vol 20, pp. 273-297, 1995

[11] Smola A J, Schölkopf B, Williamson R C, "New support vector algorithms", J, Neural Computation, , vol 12, pp. 1207-1245, 2000.

[12] G. Zhang, J Zhou, W Guo. "A Method to Reduce Samples for Support Vector Machines", C, International Conference on Virtual Reality \&Virsualization. pp.248-253.2014. 